\title{
Inhaled corticosteroids and the growth of lung function in children
}

\author{
M. Silverman
}

This month's edition of the European Respiratory Journal contains two papers that address the important topic of the effect of inhaled corticosteroids (ICS) on lung development in childhood [1, 2]. The papers are important because: 1) they raise important methodological issues concerning the design of outcome studies in children; 2) they illustrate different approaches to the measurement of lung function in different age groups (preschool children and teenagers, respectively); 3) they raise questions about the relationship between the structure and function in the developing lung; and 4) they fuel the debate about the appropriate choice of outcomes in managing ICS therapy in children. These points, in turn, are considered below.

First, the design of the two studies is quite different. Devulapalli et al. [1] carried out a longitudinal observational study of a population sample in which young children who were prescribed ICS for "recurrent bronchial obstruction" (undefined) were compared with untreated symptomatic and symptom-free groups. Although the design is justified as being "based upon ... real life", it suffers from the shortfall that an independent effect of ICS on functional outcome cannot be deduced from the results presented. At best, it is possible to conclude that both treated and untreated young children with recurrent bronchial obstruction appear functionally to return towards normal by 2 yrs of age. Multivariate analysis might have added strength to the assumption that this process was indeed related in someway to ICS therapy, rather than being simply a reflection of the natural history of the disorder.

In contrast, the study by MERKus et al. [2] was a spin-off from an influential randomised controlled trial (RCT) of ICS in childhood asthma [3]. It is a testimony to their record keeping skills that this group analysed the data some $10 \mathrm{yrs}$ later! The results of this small study are more secure, since the experimental (RCT) design does allow the causal role of ICS in the growth of lung function in children with "classical asthma" to be determined with less likelihood of bias. The conclusion was that ICS improved the post-bronchodilator patency of large and intermediate airways to close-to-normal over the 2-3 yr period of the study, but that peripheral airway function remained subnormal. The results are strengthened by the comparison of this data, with longitudinal data collected at about the same time on a healthy cohort of teenagers $[4,5]$. Given this evidence of efficacy, a "real-life" study of the overall effectiveness of ICS therapy might be the next logical step.

Secondly, the differences in approach to lung function measurement between the two studies $[1,2]$ are instructive. There are few practicable techniques for measuring lung function in nonsedated infants and toddlers. The method

Children's Asthma Centre and Institute for Lung Health, University of Leicester, Leicester, UK

Correspondence: M. Silverman, Children's Asthma Centre and Institute for Lung Health, University of Leicester, Leicester Royal Infirmary, Leicester LE2 7LX, UK. Fax: 44 1162523262. E-mail: ms70@le.ac.uk employed by Devulapalli et al. [1] relies on the observation that the tidal expiratory flow pattern varies according to the severity of airway obstruction in both adults [6] and infants [7]. In the presence of airway obstruction, the peak tidal expiratory flow is reached relatively earlier in expiration than normal, perhaps because the end-inspiratory diaphragmatic "brake" on early expiration is removed more rapidly [8]. The resulting index, the ratio of the time to peak tidal expiratory flow (TPTEF) to expiratory time $(T \mathrm{E})$, a numerical descriptor of the tidal flow pattern, is therefore, lower in the presence of airway obstruction. However, the measurement is only distantly related to airway function, and represents the output of the whole respiratory system in its neuromechanical complexity. There may be advantages in looking at the therapeutic response of the respiratory system as a whole, rather than trying to reduce the measurement of outcome to its potential components. However, in a complex system, the results should not be interpreted in terms of component processes without good evidence.

MERKus et al. [2] was able to measure lung function in some detail in their teenage group. As with the Norwegian study, they point out the importance of taking measurements after an adequate dose of a $\beta_{2}$-agonist in children with reversible airway disease. However, unlike Devullapalli et al., their healthy controls were not similarly dosed. MERKUS et al. [2] comment on the importance of normalising airway function measurement by lung size (total lung capacity) rather than by the less stable vital capacity, which is subject to bias from sex differences and pubertal growth effects. Their results are a more direct reflection of airway function than the tidal flow measurements in the study by Devulapalli et al. [1]. Does this provide for a more accurate description of lung growth?

Thirdly, growth in lung function is assumed to be an adequate surrogate for structural lung growth. It seems reasonable to assume that if lung growth is abnormal, lung function must be abnormal too, but this is not necessarily true. For instance, the two major variable determinants of maximum expiratory flow at wave speed, airway luminal calibre and airway wall elastance, could work in opposite directions to permit "normal" flow, despite reduced calibre and increased elastance [9]. Conversely, in asthma, highly abnormal and variable lung function may be completely reversible, and may not necessarily imply any permanent underlying structural abnormality.

However, this editorial begs a definition of "lung growth". It may not realistically be possible to separate structure from function in life. Does this matter? In the complexity of the respiratory system, structure and function are intimately interconnected. Hence, there are limits to the ability to imply structural growth from lung function tests. Therefore, for measuring short-term clinical outcomes and for predicting the lifelong prognosis of childhood respiratory illness, functional lung growth is likely to remain a yardstick.

Fourthly, do these two studies provide clinical guidance on the choice of outcome measures for ICS therapy in treating 
children with airway diseases? In the era of evidence-based medicine, observational studies, such as the study by Devulapalli et al. [1] contribute little. This is unfortunate, since objective experimental RCT data must be tempered by "real life" observation if it is to be realistically used in clinical practice. Does the RCT experimental design [2] help to guide clinical practice? The answer is again a cautious "No". The study certainly demonstrates some of the valuable effects (on large airway function) and limitations (on peripheral airway function) of ICS, but does not provide information on whether in the long-run, these are better therapeutic targets than symptoms or airway inflammatory markers. It is salutary to recall that in the follow-up of their trial, the Dutch group [10] showed that the gains produced by regular ICS therapy were mostly lost within 6 months of withdrawing treatment [10].

Several components are required to create a research programme to investigate the long-term risks and benefits of inhaled corticosteroids on lung growth in children. Some have been successfully used in the two studies reported here [1, 2]. They include: a carefully collected prospective cohort, ideally recruited during foetal life that will allow pre-existing risk factors for lung growth to be evaluated; a randomised controlled trial format, to avoid selection bias in relation to the intervention (inhaled corticosteroids); carefully developed, normalised lung function tests that mirror as closely as possible the functional development of the lungs during childhood and puberty; meticulous, long-term record keeping; and a commitment from funding bodies to long-term support!

\section{References}

1. Devulapalli CS, Haaland G, Pettersen M, Carlsen KH, Lodrup Carlsen K. Effect of inhaled steriods on lung function in young children: a cohort study. Eur Respir $J$ 2004; 23: 869-875.

2. Merkus PJFM, van Pelt W, van Houwelingen $\mathrm{J}$, et al. Inhaled corticosteroids and growth of airway function in asthmatic children. Eur Respir J 2004; 23: 861-868.

3. van Essen-Zandvliet EE, Hughes MD, Waalkens HJ, Duiverman EJ, Pocock SJ, Kerrebijn KF. Effect of 22 months of treatment with inhaled corticosteroids and/or $\beta-2$ agonists on lung function, airway responsiveness, and symptoms in children with asthma. The Dutch Chronic Non-specific Lung Disease Study Group. Am Rev Respir Dis 1992; 146: 547-554.

4. Borsboom GJJM, van Pelt W, Quanjer PH. Pubertal growth curves of ventilatory function: relationship with childhood respiratory symptoms. Am Rev Respir Dis 1993; 147: 372-378.

5. Merkus PJ, Borsboom GJ, van Pelt W, et al. Growth of airways and air spaces in teenagers is related to sex but not to symptoms. J App Physiol 1993; 75: 2045-2053.

6. Morris MJ, Lane DJ. Tidal expiratory flow patterns in airflow obstruction. Thorax 1981; 36: 135-142.

7. Benoist MR, Brouard JJ, Rufin P, Delacort C, Waernessyckle S, Scheinmann P. Ability of new lung function tests to assess metacholine-induced airway obstruction in infants. Pediatr Pulmonol 1994; 18: 308-316.

8. Clarke J, Silverman M. Infant lung function and tidal breathing patterns. Pediatr Pulmonol 1995; 20: 135-136.

9. Brackel HJ, Pedersen OF, Mulder PG, Overbeek SE, Kerrebijn KF, Bogaard JM. Central airways behave more stiffly during forced expiration in patents with asthma. $\mathrm{Am}$ $J$ Respir Crit Care Med 2000; 162: 896-904.

10. Waalkens HJ, van Essen-Zandvliet EE, Hughes MD, et al. Cessation of Long-term treatment with inhaled corticosteroid (budesonide) in children with asthma results in deterioration. The Dutch CNSLD Study Group. Am Rev Respir Dis 1993; 148: 1252-1257. 